\title{
A mother-daughter relationship
}

Radial glia in the ventricular zone (VZ) of the developing cortex divide asymmetrically, producing a selfrenewing radial glial cell that remains in the VZ and a cell that differentiates into a neuron or an intermediate progenitor. How these differing fates are determined was unknown; however, research from Shi and colleagues suggests that asymmetrical inheritance of centrosome components is crucial.

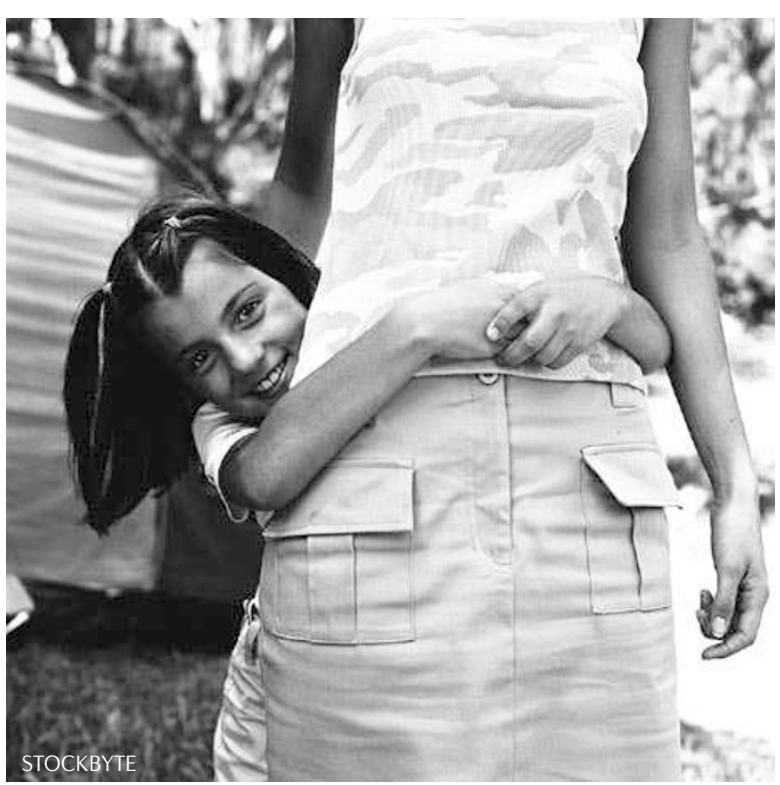

The centrosome has at its core a pair of centrioles, of which one (the 'mother' centriole) is older than the other. During mitosis, the centrioles are duplicated and the two resulting centrosomes - each consisting of one of the cell's original centrioles and one newly synthesized centriole - are distributed to the two daughter cells. This pattern of segregation means that only one daughter cell inherits the original, older mother centriole. Could the ages of the centrioles inherited by the daughter cells have a role in determining their subsequent development?

The authors labelled the centriole component centrin 1 with a protein that can be converted, by violet light exposure, from one that emits green fluorescence to one that emits red fluorescence in the brains of mice at embryonic day 13.5. Following photoconversion and two rounds of mitosis, most cells containing red centrin 1 (centrin 1 that existed at the time of photoconversion) were located in the $\mathrm{VZ}$ and co-expressed PAX6, a radial glial marker. By contrast, most cells containing only green (newly synthesized) centrin 1 had migrated out of this region and co-expressed a neuronal marker. This suggested that the older mother centriole was preferentially inherited by the daughter cell that became a self-renewing progenitor.

To determine whether this pattern of inheritance governs the daughter cell's fate, the authors used RNA interference to suppress the expression of ninein - a mother centriole-specific protein. This prevented the asymmetrical inheritance of the centrosomes and resulted in the depletion of cells from the VZ.

These findings suggest that asymmetric centriole inheritance is crucial for the maintenance of the pool of self-renewing radial glia during neurogenesis. The mechanisms by which inheritance of an older mother centriole promotes a stem cell fate are unknown; however, examining the properties of these centrioles and the proteins associated with them may provide clues.

Katherine Whalley

ORIGINAL RESEARCH PAPER Wang, X. et al. Asymmetric centrosome inheritance maintains neural progenitors in the neocortex. Nature $\mathbf{4 6 1}$, 947-955 (2009) 